\title{
Implementing and adapting the WRON-RM Use Case categories for eReefs: aiming for Interoperable Systems' requirements analysis best practice
}

\author{
$\underline{\text { N.J. Car }}^{\mathrm{a}}$, N. Murray ${ }^{\mathrm{a}}$ \\ ${ }^{a}$ CSIRO Land and Water \\ Email:nicholas.car@,csiro.au
}

\begin{abstract}
Multiple models, data services, sensor networks and other information tools are being embedded within complex and sometimes distributed architectures known as information platforms. These information platforms are increasingly being used to model multi-faceted environmental phenomena with one example being the eReefs project which will generate a near real-time view of lagoon water quality for the Great Barrier Reef. Not only may they involve many subsystems which must interoperate but they need to be able to evolve over time as new computational elements, sensor resources, models and datasets become available or cease to exist - for example a satellite's life ending.
\end{abstract}

To cater for both interoperability and evolution, system design must enable Use Cases - certain system behaviour - beyond those typically expected by end users such as data discovery and use. Use Cases for system maintenance, new subsystem addition, subsystem deprecation and overall information platform augmentation must be envisaged, articulated and catered for. For institutionally distributed information platforms such as eReefs, overall management and governance Use Cases are also of particular importance.

The Water Resources Observation Network's Reference Model (WRON-RM) provides the primary design framework for eReefs and it specifies six categories of Use Cases relevant to information platforms such as eReefs which were:

1. End User - processes of accessing information from an information platform

2. Data Provision - processes of contributing data to an information platform

3. Functionality Provision - processes of contributing data processing services

4. Enablement and Governance - processes of controlling various components

5. Cross-business Domain Integration - processes enabling integration with external systems

6. System Maintenance - processes associated with maintaining an information platform

The WRON-RM is both incomplete and untested with its preface stating: "It was recognised that Use Cases described in the document did not fully reflect the WRON and that the true Use Cases for the WRON were not well understood. As such, considerable work was going to be necessary to discover, describe and analyse these Use Cases in order to understand their impact on the requirements of the WRON."

This paper details how, by designing eReefs, some of that required work has taken place and how through building a concrete implementation of the WRON-RM, the extent to which the categorisation of Use Cases has helped the project. Specifically we relate: how Use Cases received from stakeholders, including most subsystem designers, fitted into the six Use Case categories; how the information platform's Scoping Study (Car et. al., 2012) attempted to express Use Cases in all 6 categories; additions and modifications that have been made to these categories as functional requirements of eReefs have become clearer; specific Use Case examples from each generic category - the WRON-RM's six and additions and finally best practice methods regarding requirements gathering and stakeholder engagement for future information platforms attempting to provider similar functionality to eReefs.

Keywords: $\quad$ Use Case analysis, interoperable systems, WRON, RM-ODP, eReefs 
Car \& Murray, Interoperable systems' requirements analysis best practice: Implementing and adapting the WRON-RM Use Case categories for eReefs

\section{INTRODUCTION}

eReefs is an information system with the goal of fundamentally changing the way the Great Barrier Reef is managed. It will bring together data, models, visualisations, reports and decision support tools in a distributed information system that delivers near real-time data on water quality. It will not be a single information and management system but instead an integrated "system of systems" that spans the areas that affect the Reef including paddock, catchment, estuary, reef lagoon and ocean. The objectives therefore of eReefs are:

- To provide integrated, comprehensive and defensible information and forecasting capability as the basis for a decision-making system for the Reef

- To create a communication tool via the eReefs visualisation layer to enable information and decisions to be conveyed to a range of different audiences for regulatory, advocacy, governance and general user/consumer purposes.

To assist in designing eReefs, the Reference Model for Open Distributed Processing (RM-ODP) was used (ISO, 1998). It specifies 5 viewpoints from which system designers consider the system being built in order to incorporate the wide range of requirements that distributed systems have. The viewpoints are Enterprise the business needs/drivers for the information system; Information - what and where information flows around the system; Computational - how the service interfaces are defined; Engineering - where the various components of the system are located physically (topology); and Technology - specific components (brands, versions) required for system functionality.

The eReefs design is further informed by the Water Resources Observation Network Reference Model (WRON-RM) (Lemon et. al., 2007) which was intended to be a domain-specific reference model for delivery of water information data and services. The WRON-RM's primary features were the use of the RM-ODP's viewpoints and the establishment of generic, "system" Use Cases in order to provide the foundation for a reference model implementation methodology. It also detailed water domain relevant specific standards choices and articulated some example Use Cases. Parts of the WRON-RM approach have been used in several CSIRO projects, however to date none have attempted to follow the WRON-RM methodology or to use it as an architectural basis for an entire system.

The use of viewpoints by the RM-ODP and then the WRON-RM helps to simplify the description of complex systems, such as eReefs, but do not provide guidance on the level of detail required in each viewpoint, nor on the completeness of the specification. This is reflected in the WRON-RM where the Enterprise viewpoint was the most developed, the Information and Computational viewpoints partially developed, and the Engineering and Technology viewpoints, which depended largely on the approaches taken within the Information and Computational viewpoints, not specified in any detail. There was a recognition that very high level Use Cases for the WRON-RM ("system evolvability") were not completely understood and that certain technical components necessary to realise the WRON-RM vision required considerably in order for more detail to be articulated. (Lemon et. al. 2007, sec. Preface).

While there are many instances of use of the RM-ODP ${ }^{1}$, there are, therefore, no examples of complete implementation of the WRON-RM (a "WRON demonstration system") or of validation of the WRON-RM methodology. However, since the eReefs project has adopted the WRON-RM as a foundation and is performing this validation as a side-effect of its implementation.

\section{BACKGROUND - THE WRON-RM APPROACH TO USE CASES}

\subsection{WRON-RM Use Case category description}

The WRON-RM Enterprise viewpoint classifies system-level Use Cases into six categories or perspectives. Elaborating on of each of these is the principle by which Use Cases are detailed. The categories are:

1. End User - end users accessing information for use. E,g. downloading data to a mobile device for marine survey purposes. These Use Cases are diverse but the easiest to elaborate as end-users and their representatives usually have a good idea of how it should behave;

2. Data Provision - provisioning a new data resource to be delivered using eReefs infrastructure. They describe the process for adding new data products and services to systems. They affect design decisions and influence system architecture;

\footnotetext{
${ }^{1}$ Just search for "RM-ODP" in your favourite search engine to find many papers and links to its use.
} 
Car \& Murray, Interoperable systems' requirements analysis best practice: Implementing and adapting the WRON-RM Use Case categories for eReefs

3. Functionality Provision - contributing data processing services to eReefs. An example is providing a user-selectable set of server-side processing tools for creating "mashups" of data, such as kriging temperature and salinity data from marine weather stations and comparing the resulting grid to predictions from hydrodynamic models;

4. Enablement and Governance - controlling various components of eReefs. These Use Cases consider the behaviour of resources accessible via a WRON implementation;

5. Cross-business Domain Integration - enabling integration with other frameworks. These Use Cases aim to provide a framework for integrating resources from external domains. In the case of eReefs this could, for example, involve ecological or sociological datasets being made available, though there remains the challenge to use these datasets in a meaningful way;

6. System Maintenance - maintaining eReefs infrastructure and processes to ensure the ongoing use, usage monitoring and maintenance of performance and reliability of the system. As higher-level requirements change, the maintenance regime also must be adaptable and must be aligned with governance practices in order that all maintenance roles and responsibilities can be assigned to particular stakeholders.

\subsection{WRON-RM Use Case categorisation effect}

The intended effect of the WRON-RM's categorisations is to broaden system designers' focus on system requirements. Without catering for the Use Cases in all of the WRON-RM's categories, its authors believed that ultimately systems designed would not deliver the core features of an interoperable system.

\section{THE EREEFS APPROACH TO USE CASES}

\subsection{Elicitation of Use Cases}

At the current stage of eReefs design and development with complete end-user engagement still to be undertaken, the eReefs project team has extracted Use Cases from a range of sources, including:

- WRON-RM categories: the generic Use Cases were decomposed into lower level Use Cases representing established and expected behaviour of distributed systems; for example, the generic "governance" Use Case incorporates provision of administrative/"normal" users and administrative groups as an expected characteristic of a multi-user system;

- Existing processes: those already implemented by existing eReefs systems. For Example, the Bureau of Meteorology's REEFTEMP sea surface temperature product;

- $\quad$ eReefs User Reference Group: a group formed to generate user stories which delivered a mixture of higher- and lower-level usage scenarios from which Use Cases were extracted. Example: "View forecast sediment plume maps". Use Cases taken from the URG are listed in the eReefs Work Package 2 Scoping Study (Car et. al., 2012);

- External systems: those that could possibly be integrated into, or provide data for, eReefs. They present as "system actors" and thus have associated Use Cases. Currently the Atlas of Living Australia (ALA) is one such external system ${ }^{2}$, TERN $^{3}$ another and $\operatorname{IMOS}^{4}$ yet another.

\section{2. eReefs Scoping Study categorisation of Use Cases}

In the eReefs Work Package 2 Scoping Study (Car et. al., 2012), the authors categorised the Use Cases from the sources listed above according to the 6 WRON-RM categories (see Tables $5 \& 6, \mathrm{p} 51 \& 52$ ). The major results from that exercise were:

- $\quad$ user-derived Use Cases all fitted into Cat.1, End User category;

- Cat. 2 Data Provision Use Cases were inherently present in some End User and other UCs;

- $\quad$ No Cat. 4 UCs Enablement \& Governance could be articulated;

\footnotetext{
${ }^{2} \mathrm{http://www.ala.org.au}$

3 http://tern.org.au

4 http://www.imos.org.au
} 
Car \& Murray, Interoperable systems' requirements analysis best practice: Implementing and adapting the WRON-RM Use Case categories for eReefs

- Cat. 6 UCs System Maintenance were very high-level and, due to the early stage of eReefs architectural design, no specific procedures could be implemented.

The general learning from the exercise is that it has not been possible to articulate high-level non End User UCs required by the WRON-RM from normal user requirements analysis. The WRON-RM does not provide a mechanism to do so either.

The details of UCs in the Enablement \& Governance categories are strongly affected by system architecture therefore the Scoping Study authors believed that no great progress can be made regarding them until at least the overall eReefs architecture had been more fully designed. The next Section covers technologies that affect the overall eReefs architecture and the architecture has, at the time of this paper's writing, been designed in enough detail to allow progress. Use Cases in the Cross-Domain Business Integration category required good knowledge of systems external to eReefs to articulate and the Scoping Study authors were not wholly confident in this knowledge, therefore only high-level UCs were given for this category.

\subsection{Technology change affecting the WRON-RM Use Case categories}

When the WRON-RM was initially developed, certain standards and capabilities we now regard as important for open, distributed processing were not well specified or were not available. Those of particular interest to eReefs are:

- Linked Open Data ${ }^{5}$ which enables data items and alternate views of them to be associated with URIs $^{6}$ and also allows links between datasets and metadata to have an intrinsic type;

- Model-driven data services that use data models to specify data services. eReefs uses an information model registry, in conjunction with an orchestration service, to find known data services, interrogate them to determine if they comply with their given data models (see Lemon, et. al., (2011) for definitions of model driven design and previously examples in the water domain);

- Ontology and ontology-based vocabulary services that deliver domain-specific and generic controlled vocabularies of terms with hierarchical relationships. They are used to support modeldriven development and automated methods of validating datasets;

- Test-driven service validation which extends the well-known software coding paradigm of testdriven design to the design of data services, metadata services and system configuration. This functionality is present in eReefs through an Orchestration Service that runs on an eReefs community central node.

\section{4. eReefs Architecture affecting the WRON-RM Use Case}

Use of these technologies listed in the previous section is incorporated into the eReefs architectural design as described in the eReefs Architecture wiki ${ }^{7}$. The two major architectural concepts that affect UC details are:

- a concept of interest communities containing a hierarchy of nodes that deliver data (Data Provider Nodes, DPNs), broker community access to and bridging between a series of DPNS (Community Central Nodes, CCNs) and consumer nodes that include portals, processing services etc. That ultimately use the data (Consumer Nodes, CNs);

- DPN structure: resources within DPNs need to present data, metadata and vocabulary services at a minimum and preferentially their information model delivered via a Feature Type Catalogue. In time, they will also need to deliver provenance services recording resources' processing history.

\section{WRON \& EREEFS USE CASE ARTICULATION EXAMPLE}

The Data Provision category of UCs is core to the interoperability and extensibility of a distributed system: system aspects that are expressly catered for in the WRON-RM. Without effective UCs here, new data (whole datasets or parts thereof) will not be able to be added to a system thus rendering it static. Considering UCs in this category are therefore of particular interest to the eReefs designers, beyond their regular interest

\footnotetext{
${ }^{5}$ http://en.wikipedia.org/wiki/Linked_Data

6 Uniform Resource Identifiers: see http://en.wikipedia.org/wiki/Uniform_Resource_Identifier for an overview and http://www.w3.org/TR/uri-clarification/ for in-depth discussion of URI make-up.

7 https://wiki.csiro.au/display/NE2I/eReefs+Architecture. Unfortunately this wiki is not publicly available. Contact the authors for partial access.
} 
Car \& Murray, Interoperable systems' requirements analysis best practice: Implementing and adapting the WRON-RM Use Case categories for eReefs

in, for example, End User UCs which are part of many regular software system design processes. The WRON-RM detailed some Data Provision UCs which, since the advent of the technology listed above and the specifics of eReefs we can now supersede with eReefs Data Provision UCs.

\subsection{WRON-RM Data Provision Use Case details}

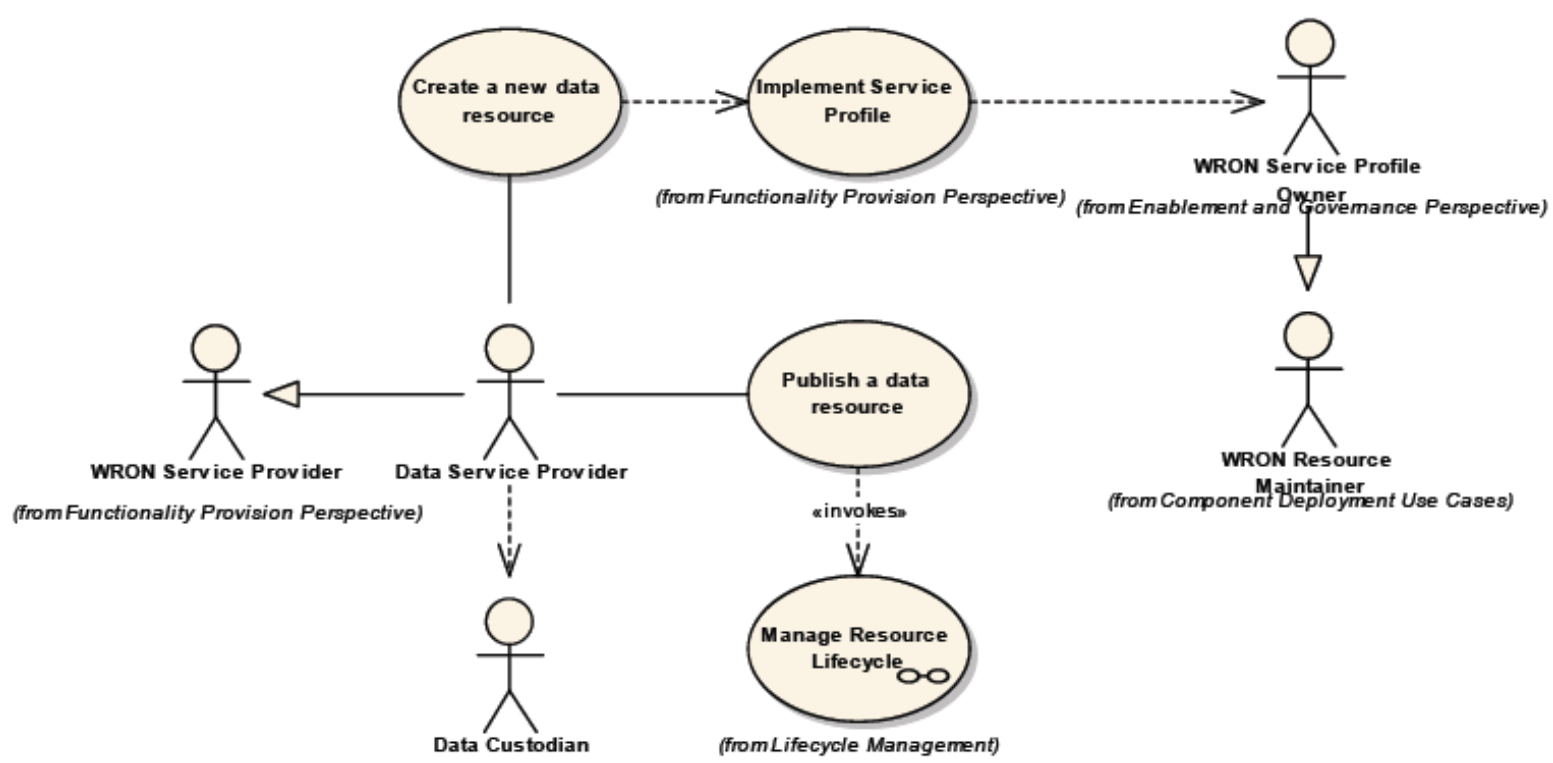

Figure 1: WRON-RM Data Provision Use Case diagram in UML

\section{2. $\quad$ eReefs Data Provision Use Case details}

Figure 1 shows a generic Data Provision category UC, according to the WRON-RM. The key aspects of this UC that afford a measure of interoperability to a system are Implement Service Profile and Publish a data resource. The first specifies what data services are needed to deliver a particular type of dataset in order for it to be compatible with a WRON-registered service profile. These service profiles specify where and how that datasets delivered as a service will be made available. These authors believe that implicit in this second point is the loading of service catalogues (metadata catalogues and others) that make the new dataset discoverable.

The eReefs interpretation of a generic Data Provision UC incorporates versions of the Implement Service Profile and Publish a data resource actions however new actions, based on the availability of new tools such as an implementation of the Test-driven Service Validation mentioned in Section 3.3, and also included.

Create a new data resource: As per the WRON-RM.

Implement a service profile: Within eReefs, the service profile will be driven by specifications surrounding eReefs Data Provider Nodes (DPN). The resource may be added to an existing DPN or have a new one created for it. Typically the resource owner will need to:

- Match new data resource metadata vocabulary terms with existing DPN metadata terms, where possible or add them if new;

- Publish the information model used for the resource via a Feature Type Catalogue or a Model Registry within the DPN;

- Create a new metadata entry data resource in the DPN's central metadata catalogue;

- Create a new persistent ID (PID) URI pattern set for the data resource in the DPN's PID service binding its newly created data, metadata and vocabulary terms together.

Publish a data resource involves:

- Initially publishing the resource solely through the resource's owning DPN, as above;

- Enabling a community's Central Node to index the resource's metadata catalogue entry (an automatic step if indexation is enabled and the implementation of the service profile is correct); 
Car \& Murray, Interoperable systems' requirements analysis best practice: Implementing and adapting the WRON-RM Use Case categories for eReefs

- Ensuring that the resource's data, metadata and vocabulary entries pass a particular community's test-driven service validation. The eReefs Orchestration Service will detect new resources made available to it through registered eReefs DPNs and will report on their validity at regular intervals;

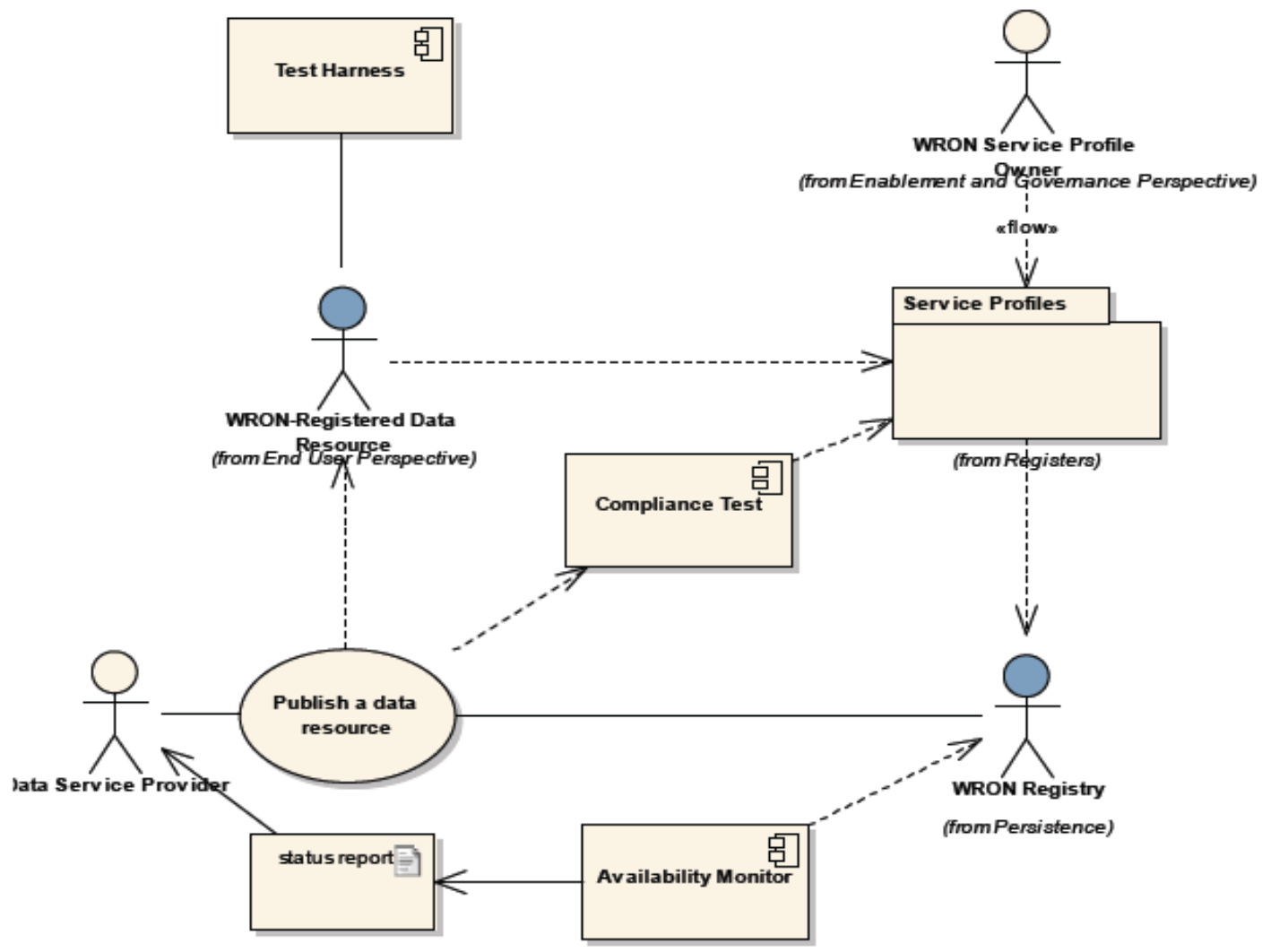

Figure 2: eReefs Data Provision Use Case diagram in UML

Manage resource lifecycle: If the resource passes initial validation (in Publish.. above), the resource owner will need to continue to ensure that the resource's components meet the eReefs required Service Level Agreement which will be continuously tested also by the eReefs Orchestration Service. Future work in this area involves recognising that datasets may only be available for a limited time period, may become deprecated (new analysis techniques used or new platform availability), or may be available intermittently. Central Node validation provides a mechanism to implement these lifecycle requirements.

\subsection{Revisiting the WRON-RM Use Case categories}

End-user: the eReefs UC elaboration process has been heavily skewed towards this category and this is likely to be the case for many projects with a strong requirement to meet non-architectural stakeholder needs.

Data provision and functionality provision: have been adapted by eReefs and are heavily dependent on new architectural design work that has been carried out since the WRON-RM's writing.

The enablement and governance, cross-business domain and maintenance categories, however, were not fully specified in the WRON-RM.

Enablement and governance did not consider the various agreements (service level agreements, memoranda of understanding, data and intellectual property sharing agreements etc.) required to be in place prior to "data provision" or "functionality provision". There are few standards supporting inter-agency aspects of interoperability standards; one attempt at such a standard is the Service Oriented Architecture Governance Interoperability framework (Hewlett-Packard, 2008). The SOA GIF attempts to promote interoperability between SOA components however it deals more with technical interaction between inter-agency components, than with interaction between agencies themselves. Technical adherence to an SLA or similar may be able to be managed through the eReefs Orchestration Service. 
Car \& Murray, Interoperable systems' requirements analysis best practice: Implementing and adapting the WRON-RM Use Case categories for eReefs

The cross-business domain: The WRON-RM did not conceive of a multi-level, nodal structure as we do now. If this nodal structure is implemented across domains, much of the work required to use resources in other domains will be able to be undertaken by the external domain. It will need to navigate a resource's PID links to its constituent services, harvest a resource's metadata, incorporate a resource's vocabulary terms in its own cached vocabulary list and run its own conformance tests (if it has an Orchestration Service) on it.

Maintenance: Maintenance is handled by requiring resources' constituent services to fit an overarching resource owner specification (the DPN) and by continuously testing their conformance and performance through communities' Orchestration Services. Tests run by these services should indicate schema validity across multiple services and within individual services and community-specific tests could be implemented by communities to test for almost anything. Base set of standardised tests the passing of which will confer recognised compliance to generic communities are also likely to be run. In this regard, eReefs resources will be tested against eReefs resource profile and the less detailed National Plan for Environmental Information resource profile which has a mandate over all Australian environmental data ${ }^{8}$.

\section{CONCLUSIONS AND RECOMMENDATIONS}

The 6 categories of UCs seem to hold utility for eReefs at the high level. The authors suggest that for the future analysis of projects similar to eReefs, Cat's $2 \& 3$ (Data Provision and Functionality Provision) be merged. This is because, due to the nodal nature of architectures like eReefs', a provided functionality presents as a resource delivered as a service, just as datasets provided do.

Since details within the Enablement \& Governance and Maintenance category UCs depend on architecture specifics, if User Reference Groups or other, similar UC elicitation processes are ever to reflect them, people within those processes need to have some understanding of proposed architecture. It is preferable that these people do reflect UCs in these other categories as often project performance metrics are tied only to "End User" requirements. This has, so far, not been the case in eReefs due to the immaturity of the architecture and the thoughts in this paper having not been formulated.

The test-driven nature of community interactions in eReefs-like projects will provide specific steps within the Enablement \& Governance and Maintenance UC categories which will apply to all eReefs-like projects. Adaptation to specific instances will be made in the particular tests used, not in a different arrangement of UCs or UC steps. This will allow a WRON-RM v2 or similar to give lots of detail for these categories.

\section{REFERENCES}

Car, N.J., Fitch, P.G. and Lemon, D. (2012) Scoping Study: eReefs Work Package 2 - Interoperable data and information systems. CSIRO, Australia. Online at https://publications.csiro.au/rpr/ download?pid=csiro:EP127142\&dsid=DS6

eReefs Collaborative Joint Venture (CJV) (2011). Collaboration Agreement in relation to the development of the eReefs system. Final copy signed, $24^{\text {th }}$ November, 2011. Unpublished.

Hewlett-Packard Development Company, L.P., (2008). Governance Interoperability Framework Reference, Online at: https://g1u1195c.austin.hp.com/cda/hpdc/navigation.do?action=downloadPDF\&caid=24529\& $\mathrm{cp}=54 \_4000 \_28 \& z n=$ bto\&filename $=4$ AA1-8129ENW.pdf

International Organization for Standardization (ISO), (1998). ISO/IEC 10746-1:1998 (E). Information technology - Open Distributed Processing - Reference model: Overview. Geneva: ISO. http://standards.iso.org/ittf/ PubliclyAvailableStandards/index.html

Lemon D, Cox S, Walker G, Atkinson R, Fitch P and O'Hagan R. 2007. Water Resources Observation Network - Reference Model Version 0.1. CSIRO: Water for a Healthy Country. 93 pp. Online at: http://www.clw.csiro.au/publications/waterforahealthycountry/2007/wfhc-WRON-Reference-ModelV0.1.pdf

Lemon, D., Atkinson, R., Box, P. and Woolf, A. (2011). The role of model driven architecture in the development of the Australian Water Resources Information System. In: WIRADA Science Symposium; 1-5 August 2011; Melbourne. CSIRO; 2011. 1p.

${ }^{8}$ http://www.environment.gov.au/npei 